in vivo $35: 229-237$ (2021)

doi:10.21873/invivo.12251

\title{
Sulfite Oxidase Is a Novel Prognostic Biomarker of Advanced Gastric Cancer
}

\author{
YUTA YANO $^{1}$, JUN AKIBA ${ }^{2}$, YOSHIKI NAITO ${ }^{2}$, EIJI SADASHIMA ${ }^{3}$, \\ ${\text { HARUHIKO } \mathrm{CHO}^{4} \text {, TSUNEKAZU HISHIMA }}^{5}$ and HIROHISA YANO ${ }^{1}$ \\ ${ }^{1}$ Department of Pathology, Kurume University School of Medicine, Fukuoka, Japan; \\ ${ }^{2}$ Department of Diagnostic Pathology, Kurume University Hospital, Fukuoka, Japan; \\ ${ }^{3}$ Life Science Research Institute, Saga-ken Medical Centre Koseikan, Saga, Japan; \\ ${ }^{4}$ Department of Surgery, Tokyo Metropolitan Komagome Hospital, Tokyo, Japan; \\ ${ }^{5}$ Department of Pathology, Tokyo Metropolitan Komagome Hospital, Tokyo, Japan
}

\begin{abstract}
Background/Aim: Sulfite oxidase (SUOX) is an enzyme present in the mitochondria, which has been demonstrated to be correlated with various malignant tumours. Materials and Methods: We evaluated SUOX expression in tissues of 98 cases of advanced gastric cancer and performed a clinicopathological assessment for metrics. Results: Among 98 cases, 55 cases were classified into the SUOX low expression group, and 43 cases into the SUOX high expression group. There were more pStage IV cases in the low expression group. The median overall survival of the low expression group was shorter than that of the high expression group $(p=0.020)$. In univariate and multivariate analysis, SUOX low expression level $(p=0.039)$ and pStage $(p<0.001)$ were independent prognostic factors. Conclusion: SUOX is an independent prognostic factor. Therefore, SUOX expression could also serve as a useful marker for elucidating the mechanism of gastric cancer proliferation and progression.
\end{abstract}

Gastric cancer is the fifth most common malignant epithelial tumour in the world and the second leading cause of tumourrelated deaths, along with liver cancer (1). In particular, the incidence rate of gastric cancer is extremely high in East Asia, where the infection rate of $H$. pylori is high (2). Following lung and colorectal cancer, gastric cancer is the cause of the highest number of deaths in Japan (3). Even so,

This article is freely accessible online.

Correspondence to: Jun Akiba, MD, Ph.D., Department of Diagnostic Pathology, Kurume University Hospital, 67, Asahimachi, Kurume-shi, Fukuoka 830-0011, Japan. Tel: +81 942353311, e-mail: akiba@med.kurume-u.ac.jp

Key Words: Gastric cancer, sulfite oxidase, prognosis, immunohistochemistry. the incidence rate of gastric cancer has been decreasing in recent years due to the elimination of $H$. pylori (4). Furthermore, the prognosis of patients with gastric cancer has been improving with detection at early stages through the establishment of effective screening systems, as well as the introduction of molecular-targeted therapeutic agents targeting human epidermal growth factor receptor 2 (HER2) for advanced gastric cancer $(5,6)$. However, the prognosis of advanced gastric cancer remains poor, and there is a strong demand for the development of effective treatments.

Cancer cells have been demonstrated to utilize glycolysis for energy production, even with sufficient environmental oxygen $(7,8)$. This lack of oxidative phosphorylation in the mitochondria is called the Warburg effect. Gastric cancer cells use this mechanism to generate energy for proliferation (7). Gastric cancer also suppresses mitochondrial function and exhibits resistance to apoptosis (9). The lack of mitochondrial function is characterized by the production of lactic acid through the promotion of glycolysis in the cytoplasm, which causes acidosis in the microenvironment, damages surrounding normal organs, and creates an environment conducive for infiltration and metastasis of cancer cells (7). Thus, it is speculated that clarification of the mechanism of ATP production is an important key to the growth and progression of cancer.

There are studies conducted on oral squamous cell carcinoma, prostate cancer, and pancreatic cancer that focus on sulfite oxidase (SUOX), an enzyme in the mitochondria, and one of the indicators of oxidative phosphorylation (1012). SUOX is an essential enzyme in organisms, and the genetic abnormality associated with loss of SUOX causes neurological abnormalities due to reduced mitochondrial function, often resulting in death at an early age (13). SUOX expression gradually decreases with tumour progression in oral squamous cell carcinoma, whereas SUOX expression increases with the proliferation and progression of prostate 
Table I. Clinicopathological findings and characteristics of patients with gastric cancer.

\begin{tabular}{lcc}
\hline & Case No. & $\%$ \\
\hline Patients & 98 & \\
Age, median (range) & $65(23-83)$ & \\
Gender & & $(64)$ \\
$\quad$ Male & 63 & $(36)$ \\
Female & 35 & \\
Histology & & $(39)$ \\
Differentiated & 38 & \\
Undifferentiated & 60 & $(11)$ \\
pT stage & & $(28)$ \\
T2 & 11 & $(61)$ \\
T3 & 27 & $(91)$ \\
T4 & 60 & \\
Lymphatic invasion & 88 & $(79)$ \\
Vascular invasion & 89 & \\
Lymph node metastasis & 77 & \\
pStage & & \\
I & 5 & $(51)$ \\
II & 28 & \\
III & 50 & $(15)$ \\
IV & 15 & \\
OS (days) median (range) & $1,080(19,6,700)$ \\
\hline
\end{tabular}

OS: Overall survival.

and pancreatic cancer (10-12). In hepatocellular carcinoma, SUOX expression decreases with gradual tumour progression (14). Thus, although SUOX is an important factor involved in tumour growth and progression, there are differences in its expression pattern depending on the carcinoma. Presently, there are no studies concerning SUOX in gastric cancer. Here, we investigated the relationship between SUOX expression in gastric cancer and clinicopathological factors including prognosis.

\section{Materials and Methods}

Patients and tissue samples. The subjects included in this study underwent surgical resections for gastric cancer at the Tokyo Metropolitan Cancer and Infectious Diseases Center Komagome Hospital (hereinafter, Komagome Hospital) from 2000 to 2001. A clinicopathological study was performed on paraffin-embedded samples of 98 cases of gastric adenocarcinoma, excluding cases that showed differentiation into neuroendocrine components and squamous cell components, mesenchymal tumours, residual gastric cancer, and perioperative deaths. Furthermore, the cases in this study had advanced cancer with a tumour depth reaching the muscularis propria ( $\geq$ pT2). For pathological diagnosis, we used the Japanese Classification of Gastric Carcinoma edited by the Japanese Gastric Cancer Association (15). We classified 98 cases into the differentiated type and undifferentiated type of adenocarcinoma. The former included papillary adenocarcinoma, tubular adenocarcinoma, well-differentiated type, tubular adenocarcinoma, and moderately differentiated type. The latter
Table II. Correlation between SUOX expression and clinicopathological characteristics.

\begin{tabular}{|c|c|c|c|c|c|}
\hline & \multicolumn{2}{|c|}{ Low expression } & \multicolumn{2}{|c|}{ High expression } & \multirow[t]{2}{*}{$p$-Value } \\
\hline & No. & $(\%)$ & No. & $(\%)$ & \\
\hline Patients & 55 & $(56)$ & 43 & $(44)$ & \\
\hline \multicolumn{6}{|l|}{ Age } \\
\hline$<65$ & 29 & (53) & 20 & (47) & \multirow[t]{2}{*}{0.515} \\
\hline$\geq 65$ & 26 & (47) & 23 & (54) & \\
\hline \multicolumn{6}{|l|}{ Gender } \\
\hline Male & 35 & (64) & 28 & $(65)$ & \multirow[t]{2}{*}{0.879} \\
\hline Female & 20 & (36) & 15 & (35) & \\
\hline \multicolumn{6}{|l|}{ Histology } \\
\hline Differentiated & 20 & (36) & 18 & $(42)$ & \multirow[t]{2}{*}{0.579} \\
\hline Undifferentiated & 35 & (64) & 25 & $(58)$ & \\
\hline \multicolumn{6}{|l|}{ pT stage } \\
\hline $\mathrm{T} 2$ & 4 & (7) & 7 & $(16)$ & \multirow[t]{3}{*}{0.157} \\
\hline $\mathrm{T} 3$ & 13 & (24) & 14 & (33) & \\
\hline $\mathrm{T} 4$ & 38 & (69) & 22 & (51) & \\
\hline Lymphatic invasion & 51 & (93) & 37 & (86) & 0.327 \\
\hline Vascular invasion & 51 & (93) & 38 & (88) & 0.500 \\
\hline Lymph node metastasis & 46 & (84) & 31 & (74) & 0.236 \\
\hline \multicolumn{6}{|l|}{ pStage } \\
\hline I & 1 & (2) & 4 & (9) & \multirow[t]{4}{*}{$<0.001$} \\
\hline II & 14 & (26) & 14 & (33) & \\
\hline III & 26 & $(47)$ & 24 & (56) & \\
\hline IV & 14 & (26) & 1 & (2) & \\
\hline $\begin{array}{l}\text { OS (days) median } \\
\text { (range) }\end{array}$ & \multicolumn{2}{|c|}{$\begin{array}{c}581 \\
(19,6,293)\end{array}$} & \multicolumn{2}{|c|}{$\begin{array}{c}1289 \\
(68,6,700)\end{array}$} & 0.020 \\
\hline $\begin{array}{l}\text { Ki-67 labeling } \\
\text { index }\end{array}$ & $\begin{array}{r}0 \\
(0.115\end{array}$ & $\begin{array}{l}3 \\
0.898)\end{array}$ & $\begin{array}{r}0 \\
(0.11\end{array}$ & $0.835)$ & 0.804 \\
\hline
\end{tabular}

OS: Overall survival.

included poorly differentiated adenocarcinoma, solid type, poorly differentiated adenocarcinoma, non-solid type, and signet ring cell carcinoma. This classification was proposed by Nakamura et al. (16) at the same time as the Lauren classification. This study was approved by the Research Ethics Committee of Komagome Hospital (\#2558) and Kurume University (\#20105), which conforms to the guidelines of the Declaration of Helsinki. Histopathological evaluations were performed using representative full-face sections of the tumours. Pathological diagnosis was performed according to the 2019 World Health Organization Classification of Digestive System Tumours (17).

Immunohistochemical analysis. In this study, immunohistochemical staining (IHC) was conducted using tissue microarray (TMA) sections. The TMA was built using an arraying instrument for assembling tissue samples on a block. We punctured paraffinembedded samples in two cores from the tumour portion. Core diameter samples measuring $2 \mathrm{~mm}$ were obtained from each "donor" block and placed on a separate "recipient" TMA block. Each slide contained 48 cores. Paraffin-embedded TMA sections were cut to a thickness of $4 \mu \mathrm{m}$, examined on a coated slide glass, and labelled with anti-SUOX antibody (dilution 1:600, Abcam, Cambridge, MA, USA) and anti-Ki-67 antibody (NCL-Ki67-MM1, dilution 1:200, Leica Biosystems, Nussloch, Germany) using a 

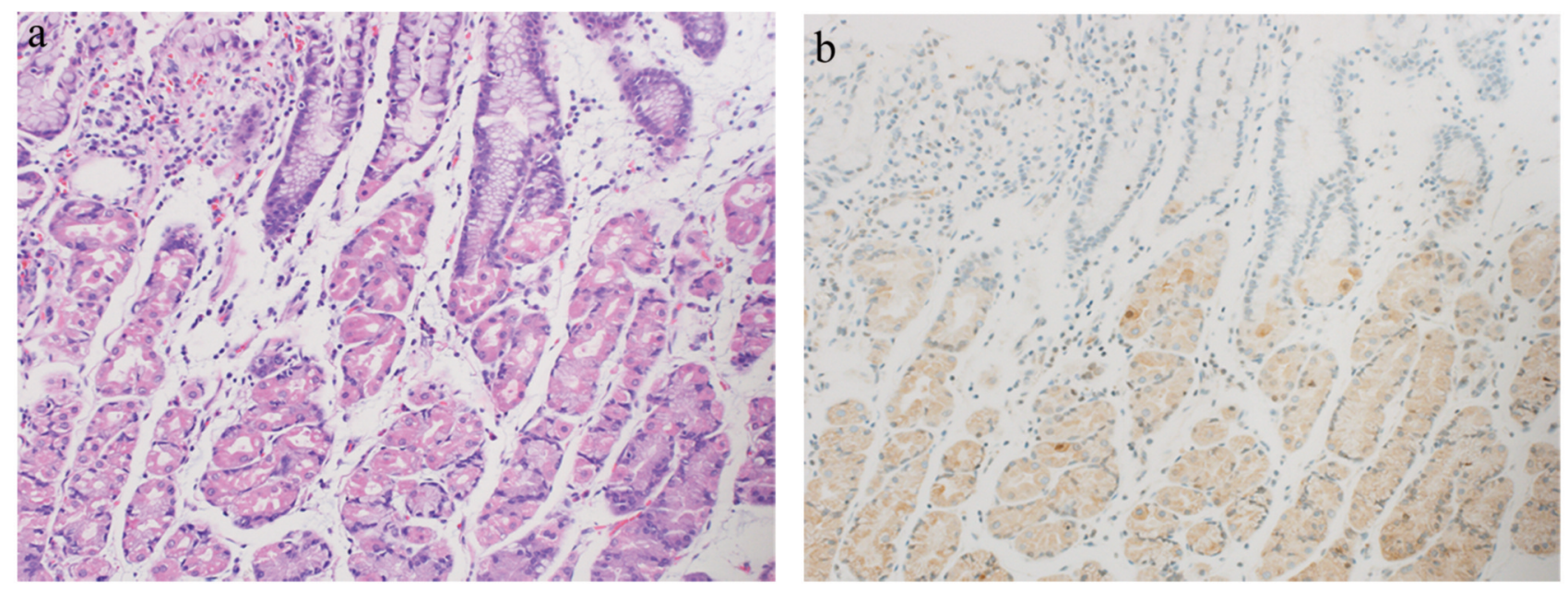

Figure 1. SUOX (sulfite oxidase) expression of non-neoplastic gastric mucosa. Photomicrographs of non-neoplastic gastric mucosa (a) stained with hematoxylin-eosin (×200), or (b) immunostained with SUOX (×200). Cytoplasmic SUOX expression was considered positive.

BenchMark ULTRA (Ventana Automated Systems, Inc., Tucson, AZ, USA). Briefly, for SUOX and Ki-67, the slide was heat-treated using Ventana's ULTRA cell conditioning retrieval solution $(\mathrm{CC} 1$, Ventana Automated Systems, Inc.) for $36 \mathrm{~min}$ and $64 \mathrm{~min}$, respectively, at $95^{\circ} \mathrm{C}$, and incubated with anti-SUOX antibody or anti-Ki-67 antibody for $32 \mathrm{~min}$ at $37^{\circ} \mathrm{C}$. The automated system used the Ventana UltraVIEW DAB detection kit with HRP (horseradish peroxidase) multimer as the secondary antibody and 3,3'diaminobenzidine as the chromogen. Each slide was incubated with a secondary antibody for $30 \mathrm{~min}$ at $37^{\circ} \mathrm{C}$.

We considered cytoplasmic expression of SUOX as positive. The percentage of positively stained neoplastic cells was also evaluated by every $10 \%$ increment. To obtain the cut-off value for SUOX, time-dependent receiver operating characteristic (ROC) curve analysis was performed. These evaluations were conducted by three pathologists (Y.Y., J.A., and Y.N.). The Ki-67 labelling index was calculated as the percentage of tumour cells that showed positive expression in the area with the highest Ki-67 expression level (hot spot). The relationship between SUOX and the clinicopathological factors was analysed in each histological type.

Statistical analysis. Data are presented as medians (ranges) or as numbers (percentages). Comparisons between groups were made using the Wilcoxon rank-sum test for continuous variables and the Fisher's exact test or chi-square test for categorical data. Spearman's rank correlation was used to analyse the relationship between two individual continuous variables. Overall survival (OS) was defined as the time from the day of surgery to death from any cause. OS was estimated using the Kaplan-Meier method. Differences in the OS rates were tested by the log-rank test. The Cox proportional hazard model was applied to evaluate the effect of SUOX while adjusting for potential confounding factors. $p$ Values less than 0.05 were considered statistically significant. All analyses were performed with the use of SAS version 9.4 (SAS, Institute Inc., Cary, NC, USA) and R version 3.4.4. Cut-off values were chosen according to the Youden index, with respect to sensitivity and specificity.

\section{Results}

Patient characteristics. The clinicopathological characteristics of the 98 gastric adenocarcinoma cases are presented in Table I. When divided according to histological types, 38 cases (39\%) were differentiated while the remaining 60 cases $(61 \%)$ were undifferentiated. The tumour infiltration depth in 11 cases $(11 \%)$ was pT2, invading the muscularis propria, and in 27 cases $(28 \%)$ was pT3, invading the subserosa. The depth in 60 cases $(61 \%)$ was pT4, perforating the serosa or invading adjacent structures. Lymph node metastasis was observed in 77 cases (79\%), lymphatic invasion in 88 cases $(90 \%)$, and venous invasion in 89 cases $(91 \%)$. The median OS was 1,080 days (range $=19-6,700$ days).

Immunohistochemical analysis of SUOX expression. SUOX positive staining was observed in the cytoplasm in the normal gastric mucosa of the gastric glands (Figure 1). Representative microscopic immunostaining images of SUOX expression and non-expression in differentiated and non-differentiated gastric cancer types are presented in Figure 2. By constructing an ROC curve, SUOX expression was graded as follows: low expression (where less than $15 \%$ of the neoplastic cells were stained) or high expression (where $\geq 15 \%$ of the neoplastic cells were stained) despite the staining intensity. The relationship between SUOX expression and various clinicopathological factors are presented in Table II. Low SUOX expression was observed in 55 of 98 cases $(56 \%)$. There was no significant difference in the degree of differentiation, tumour depth ( $\mathrm{T}$ factor), lymph node metastasis ( $\mathrm{N}$ factor), lymphatic invasion, or venous invasion between the low expression and high expression groups. Moreover, there was no correlation between 



Figure 2. Microscopic findings of representative cases of advanced gastric carcinoma (a, c, e, and $g$ ) stained with hematoxylin-eosin ( $\times 200)$, or (b, $d, f$, and $h$ ) immunostained with SUOX (sulfite oxidase) ( $\times 200)$. Cytoplasmic SUOX expression was considered high $(b$ and $f)$ or low $(d$ and $h)$.

SUOX expression and Ki-67 labelling index. Nevertheless, there were many pStage IV cases in the SUOX low expression group, and a significant difference was observed in the number of cases in each pStage between the two groups $(p<0.001)$. The low expression group had a significantly shorter median OS of 581 days (range=19-6,293 days) compared to the high expression group with a median of 1289 days (range $=68-6,700$ days) $(p=0.020)$. Kaplan-Meier curves demonstrated that the OS was significantly shorter in patients with low SUOX expression than in those with high SUOX expression $(p=0.040)$ (Figure 3).
Univariate and multivariate analysis for overall survival. The results of univariate and multivariate analysis for OS are presented in Table III. In the univariate analysis, pStage (1, 2 vs. 3, 4; $p<0.001$ ), depth (pT4 vs. pT2, pT3; $p<0.001$ ), lymph node metastasis (present vs. absent; $p=0.001$ ), lymphatic invasion (present $v s$. absent; $p=0.024$ ), venous invasion (present $v s$. absent; $p=0.032$ ), and SUOX expression $(>15 \%$ vs. $\leq 15 \% ; p=0.039)$ were extracted as prognostic factors. In the multivariate analysis, SUOX (>15\% vs. $\leq 15 \%$; $p=0.039)$ and pStage $(3,4 v s .1,2 ; p<0.001)$ were the only independent prognostic factors. 
Comparison of SUOX expression and histological features in gastric cancer. The relationship between SUOX expression and clinicopathological factors was explored in 38 cases of differentiated gastric cancer (Table IV) and 60 cases of undifferentiated gastric cancer (Table V). In the differentiated type, 20 cases (53\%) were included in the SUOX low expression group and 18 cases (47\%) in the high expression group. There was no significant difference between the two groups, even when cases were classified according to the pStage $(p=0.675)$. There were no significant differences in the $\mathrm{T}$ factor and $\mathrm{N}$ factor caused by pStage ( $p=0.137$ and $p=1.000$, respectively). There was also no significant correlation between the SUOX expression and Ki-67 labelling index in any of the histological types. However, the SUOX low-expression group in the differentiated type had a significantly shorter OS of 493 days (range $=19-2,277$ days) compared to the OS of 2,321 days (range $=86-6,085$ days) $(p=0.003)$ of the high expression group. Kaplan-Meier curves showed a similarly significant difference (log rank test: $p=0.050$ ) (Figure 4a).

In the undifferentiated group, 35 cases $(58 \%)$ were in the low expression group, and 25 cases (42\%) were in the high expression group. Among the 15 Stage IV cases, 13 cases $(87 \%)$ were in the SUOX low expression group. Despite this, there were no significant differences between the SUOX low expression group and high expression group. Similarly, Kaplan-Meier curves showed no significant difference (log rank test: $p=0.300$ ) (Figure $4 b$ ).

Moreover, the cases with high SUOX expression in the differentiated group of gastric cancers had a better prognosis than those with low SUOX expression in the differentiated group, as well as those in the undifferentiated type gastric cancers (log-rank test: $p=0.200$ ) (Figure 4c).

\section{Discussion}

In this study, we conducted an immunohistochemical analysis to examine SUOX expression in a TMA section of 98 cases of advanced gastric cancer and closely examined the relationship between SUOX expression and clinicopathological factors. Low expression of SUOX was observed in $56 \%$ of the examined cases, and cases with low expression correlated with advanced pStage. Low expression of SUOX was found to be an independent prognostic factor, along with pStage and vascular invasion. Further, we demonstrated that cases with high SUOX expression in the differentiated type of gastric cancer showed better prognosis.

This study showed that the low expression of SUOX in gastric cancer was associated with advanced stage and shorter OS. It is worth noting that cases of stage IV advanced cancer were mostly in the SUOX low expression group and had a poorer prognosis than the SUOX high-expression group. In gastric cancers characterized by multiple pathogeneses, the cross-sectional prognostic factors varied depending on the

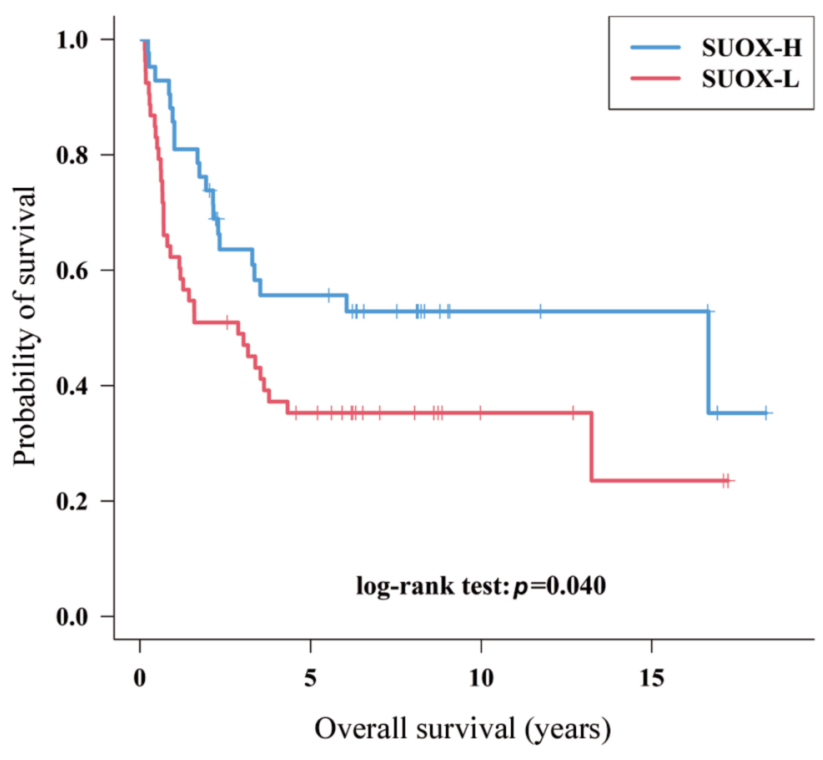

Figure 3. Kaplan-Meier curve comparing groups of high and low SUOX (sulfite oxidase) expression. The overall survival was significantly shorter in patients with low SUOX expression than in those with high SUOX expression $(p=0.040)$. SUOX-H: SUOX high expression group; SUOX-L: SUOX low expression group.

report (18-20). Our study found no association between SUOX expression and clinicopathological factors such as vascular invasion and depth of invasion. The fact that SUOX expression along with pStage were identified as independent poor prognostic factors in the multivariate analysis suggests that changes in the mechanism of energy production in gastric cancer are one of the factors that influence tumour aggressiveness.

The validity of this result is also affirmed by the presence of the Warburg effect, which promotes ATP production by glycolysis, rather than mitochondrial oxidative phosphorylation, under aerobic conditions. It is also possible that various factors encouraging tumour growth due to the Warburg effect act in combination, making low SUOX expression a poor prognostic factor. We examined SUOX protein expression immunohistochemically; however, from a comprehensive perspective, it is possible that we have indirectly evaluated the decrease in mitochondrial function due to the Warburg effect by examining SUOX expression. In our previous studies, the relationship between SUOX expression level and aggressiveness differed depending on the type of carcinoma (10-12). In other words, the activity of SUOX may vary depending on the carcinoma. It is possible that the data presented here show a difference in the balance between the inhibition of mitochondrial function by the Warburg effect and the energy production of glycolysis in the cytoplasm. Further studies are still needed to elucidate this mechanism. 
Table III. Univariate and multivariate analyses for overall survival.

\begin{tabular}{|c|c|c|c|c|}
\hline \multirow[t]{2}{*}{ Characteristic } & \multicolumn{2}{|c|}{ Univariate } & \multicolumn{2}{|c|}{ Multivariate } \\
\hline & $\operatorname{HR}(95 \% \mathrm{Cl})$ & $p$-Value & $\mathrm{HR}(95 \% \mathrm{Cl})$ & $p$-Value \\
\hline SUOX $(\leq 15 \% />15 \%)$ & $1.780(1.026,3.088)$ & 0.04 & $1.820(1.031,3.214)$ & 0.039 \\
\hline Age $(\geq 65 /<65)$ & $1.104(0.649,1.878)$ & 0.716 & & \\
\hline Gender $(\mathrm{F} / \mathrm{M})$ & $0.939(0.541,1.629)$ & 0.822 & & \\
\hline Histology (UT/DT) & $1.134(0.649,1.980)$ & 0.659 & & \\
\hline pStage $(3,4 / 1,2)$ & $6.852(3.046,15.41)$ & $<0.001$ & $6.485(2.696,15.601)$ & $<0.001$ \\
\hline pT stage $(\mathrm{T} 4 / \mathrm{T} 2, \mathrm{~T} 3)$ & $3.411(1.819,6.394)$ & $<0.001$ & & \\
\hline Lymphatic invasion & $5.102(1.238,21.03)$ & 0.024 & $1.211(0.208,7.054)$ & 0.832 \\
\hline Vascular invasion & $4.696(1.138,19.38)$ & 0.032 & $3.020(0.551,16.542)$ & 0.203 \\
\hline LN metastasis & $5.432(1.952,15.12)$ & 0.001 & & \\
\hline Ki-67 labeling index (10\% increments) & $1.040(0.900,1.201)$ & 0.599 & & \\
\hline
\end{tabular}

F: Female; M: male; UT: undifferentiated type; DT: differentiated type; LN: lymph node.

Table IV. Correlation between SUOX expression and clinicopathological characteristics in the differentiated type.

\begin{tabular}{|c|c|c|c|c|c|}
\hline \multirow[t]{2}{*}{ Differentiated type } & \multicolumn{2}{|c|}{ Low expression } & \multicolumn{2}{|c|}{ High expression } & \multirow[t]{2}{*}{$\begin{array}{c}\mathrm{n}=38 \\
p \text {-Value }\end{array}$} \\
\hline & No. & $(\%)$ & No. & $(\%)$ & \\
\hline Patients & 20 & (53) & 18 & $(47)$ & \\
\hline \multicolumn{5}{|l|}{ pT stage } & 0.803 \\
\hline $\mathrm{T} 2$ & 2 & $(10)$ & 3 & (17) & 0.137 \\
\hline $\mathrm{T} 3$ & 6 & $(30)$ & 10 & $(55)$ & \\
\hline $\mathrm{T} 4$ & 12 & $(60)$ & 5 & $(28)$ & \\
\hline Lymphatic invasion & 17 & $(85)$ & 15 & (83) & 1.000 \\
\hline Vascular invasion & 19 & (95) & 15 & (83) & 0.328 \\
\hline $\begin{array}{l}\text { Lymph node metastasis } \\
\text { pStage }\end{array}$ & \multicolumn{4}{|c|}{ pStage } & 1.000 \\
\hline I & 1 & (5) & 1 & (6) & 0.675 \\
\hline II & 6 & (30) & 9 & $(50)$ & \\
\hline III & 12 & $(60)$ & 7 & (39) & \\
\hline IV & 1 & (5) & 1 & (6) & \\
\hline $\begin{array}{l}\text { OS (days) median } \\
\text { (range) }\end{array}$ & \multicolumn{2}{|c|}{$\begin{array}{c}493 \\
(19,2277)\end{array}$} & \multicolumn{2}{|c|}{$\begin{array}{c}2321 \\
(86,6085)\end{array}$} & 0.003 \\
\hline $\begin{array}{l}\text { Ki-67 labeling } \\
\text { index }\end{array}$ & \multicolumn{2}{|c|}{$\begin{array}{c}0.566 \\
(0.145,0.754)\end{array}$} & \multicolumn{2}{|c|}{$\begin{array}{c}0.678 \\
(0.218,0.835)\end{array}$} & 0.091 \\
\hline
\end{tabular}

OS: Overall survival.

Gastric cancers comprise a series of tumours with histologically diverse pathological findings, various clinicopathological characteristics, and varied prognoses (2124). The Lauren classification, which is most widely used internationally, has broadly classified gastric cancers into intestinal type and diffuse type. In Japan, however, the Japanese Classification of Gastric Carcinoma has widely classified gastric cancer cases into differentiated type and undifferentiated type. The differentiated type and undifferentiated type in the Japanese Classification correspond
Table V. Correlation between SUOX expression and clinicopathological characteristics in the undifferentiated type.

\begin{tabular}{|c|c|c|c|c|c|}
\hline \multirow[t]{2}{*}{ Undifferentiated type } & \multicolumn{2}{|c|}{ Low expression } & \multicolumn{2}{|c|}{ High expression } & \multirow{2}{*}{$\begin{array}{r}\mathrm{n}=60 \\
p \text {-Value }\end{array}$} \\
\hline & No. & $(\%)$ & No. & $(\%)$ & \\
\hline Patients & 35 & $(58)$ & 25 & (42) & \\
\hline Age median (range) & $61(51-70)$ & & $68(28-82)$ & & 0.113 \\
\hline \multicolumn{6}{|l|}{ pT stage } \\
\hline $\mathrm{T} 2$ & 2 & (6) & 4 & (16) & 0.447 \\
\hline $\mathrm{T} 3$ & 7 & (20) & 4 & (16) & \\
\hline $\mathrm{T} 4$ & 26 & (74) & 17 & (68) & \\
\hline Lymphatic invasion & 34 & $(97)$ & 22 & $(88)$ & 0.298 \\
\hline Vascular invasion & 35 & $(100)$ & 25 & $(100)$ & 1.000 \\
\hline Lymph node metastasis & 31 & $(89)$ & 17 & (71) & 0.102 \\
\hline \multicolumn{6}{|l|}{ pStage } \\
\hline I & 0 & (0) & 3 & (12) & $<0.001$ \\
\hline II & 8 & (23) & 5 & (20) & \\
\hline III & 14 & (40) & 17 & (68) & \\
\hline IV & 13 & (37) & 0 & (0) & \\
\hline $\begin{array}{l}\text { OS (days) median } \\
\text { (range) }\end{array}$ & \multicolumn{2}{|c|}{$\begin{array}{c}1156 \\
(51,6393)\end{array}$} & \multicolumn{2}{|c|}{$\begin{array}{c}852 \\
(68,6700)\end{array}$} & 0.393 \\
\hline $\begin{array}{l}\text { Ki-67 labeling } \\
\text { index }\end{array}$ & \multicolumn{2}{|c|}{0.613} & \multicolumn{2}{|c|}{$\begin{array}{c}0.488 \\
(0.113 .0765)\end{array}$} & 0.096 \\
\hline
\end{tabular}

OS: Overall survival.

to the intestinal type and diffuse type in the Lauren classification, respectively. Although there have been varying reports regarding the prognosis of intestinal and diffuse types of gastric cancer, a meta-analysis of more than 60,000 cases has shown that the diffuse type has a significantly poor prognosis (25). Nevertheless, it has been pointed out that this classification does not allow individualization of the treatment of patients (26). That being said, in the past 20 years or so, great strides have been made in the molecular analysis of gastric cancer, leading to the emergence of several molecular 
a

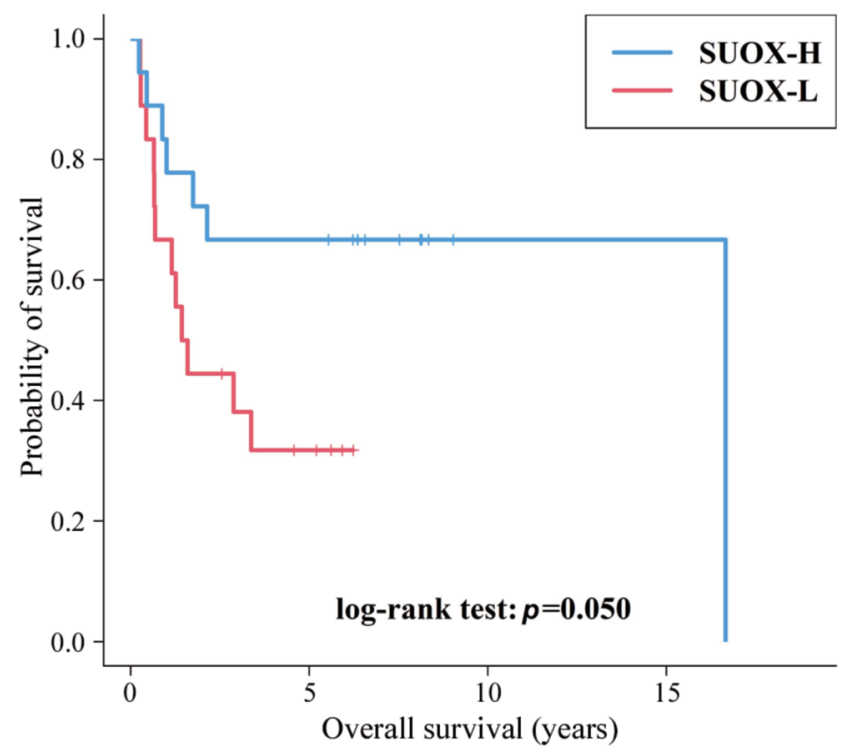

b

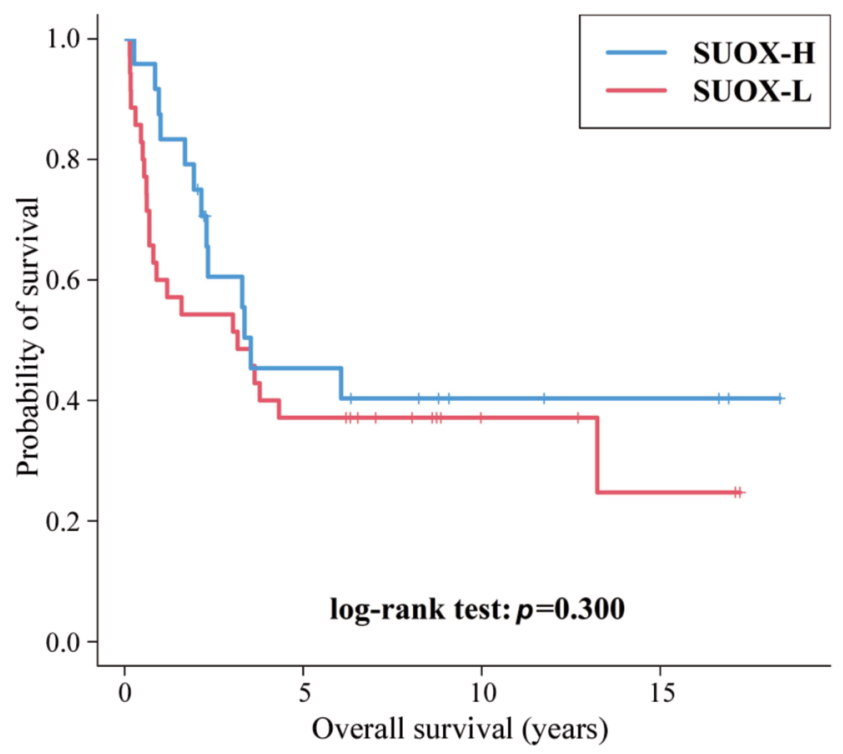

\section{C}



Figure 4. Kaplan-Meier curve comparing SUOX (sulfite oxidase) expression (low and high) and histological types (differentiated and undifferentiated). DT: Differentiated type; UDT: undifferentiated type; SUOX-H: SUOX high expression group; SUOX-L: SUOX low expression group.

pathological markers, such as Her-2/neu and c-erbB2 $(5,24)$. Furthermore, comprehensive genetic analyses in recent years have enabled the classification of gastric cancers into EBVrelated gastric cancer, gastric cancers with microsatellite instability, cancers with chromosomal instability (CIN), and genetically stable (GS) cancers (21). It was clarified that most of the histological types of cancer showing CIN correspond to the intestinal type (differentiated type), and most of the cancers classified as GS correspond to the diffuse type (undifferentiated type). Other features of gastric cancer showing CIN include TP53 mutation, activation of receptor tyrosine kinases (RTKs), and RAS and PI3-kinase signalling. In this study, the cases with high SUOX expression in the differentiated group of gastric cancers had a better prognosis than those with low SUOX expression in the differentiated group, as well as those in the undifferentiated type gastric cancers. There was also no association with clinicopathological factors such as vascular invasion and depth of invasion. 
Additionally, it has been demonstrated that p53 is involved in the expression of cytochrome $c$ oxidase 2 (SCO2) synthesis, which is an important regulator of the cytochrome c complex needed in mitochondrial respiration, and that SCO2 expression decreases in p53-deficient cells, resulting in increased energy production by glycolysis (27). Based on these facts, it is possible that cases with TP53 mutation were enriched among cases with SUOX low expression in the differentiated type, and that the decrease in mitochondrial function resulted in poor prognosis even in cases with similar histological types. To confirm this and elucidate a mechanism of regulation, a study that includes TP53 status is necessary.

There are several limitations of this study. This study did not evaluate the invasion front, which has a high proliferative activity. There has previously been a report showing the relationship between the Ki-67 positivity rate and prognosis in gastric cancer (28); however, we were unable to find any association between Ki-67 and SUOX expression due to the use of TMA sections. Additionally, as all of the samples used in this study are cases of advanced gastric cancer, cases of early stage cancer were not examined. We believe that understanding the dynamics of SUOX in early cancer will thoroughly clarify the role of SUOX in gastric cancer.

In conclusion, this study has demonstrated that SUOX is an independent prognostic factor in gastric adenocarcinoma, and that the SUOX low expression group in the differentiated type has a poor prognosis. Based on these results, we conclude that SUOX is a novel biomarker for extracting the poor prognosis group from differentiated gastric cancer, which was previously thought to have a relatively good prognosis. Therefore, SUOX could also serve as a useful marker for elucidating the mechanism involved in the proliferation and progression of gastric cancer.

\section{Conflicts of Interest}

All Authors have declared no conflicts of interest regarding this study.

\section{Authors' Contributions}

YY, JA and YN designed this study. YY drafted the manuscript, and JA, YN and HY edited the manuscript. YY, HC and TH acquired and collected the data. ES performed the statistical analysis and drew the essential diagrams.

\section{References}

1 Bray F, Ferlay J, Soerjomataram I, Siegel RL, Torre LA and Jemal A: Global cancer statistics 2018: GLOBOCAN estimates of incidence and mortality worldwide for 36 cancers in 185 countries. CA Cancer J Clin 68(6): 394-424, 2018. PMID: 30207593. DOI: $10.3322 /$ caac. 21492
2 Fock KM and Ang TL: Epidemiology of Helicobacter pylori infection and gastric cancer in Asia. J Gastroenterol Hepatol 25(3): 479-486, 2010. PMID: 20370726. DOI: 10.1111/j.14401746.2009.06188.x

3 PCancer Registry and Statistics in Japan, 2018. Available at: https://ganjoho.jp/reg_stat/statistics/stat/summary.html [Last accessed August 27, 2020]

4 Lee YC, Chiang TH, Chou CK, Tu YK, Liao WC, Wu MS and Graham DY: Association between helicobacter pylori eradication and gastric cancer incidence: A systematic review and metaanalysis. Gastroenterology 150(5): 1113-1124.e1115, 2016. PMID: 26836587. DOI: 10.1053/j.gastro.2016.01.028

5 Ajani JA, Lee J, Sano T, Janjigian YY, Fan D and Song S: Gastric adenocarcinoma. Nat Rev Dis Primers 3: 17036, 2017. PMID: 28569272. DOI: 10.1038/nrdp.2017.36

6 Bang YJ, Van Cutsem E, Feyereislova A, Chung HC, Shen L, Sawaki A, Lordick F, Ohtsu A, Omuro Y, Satoh T, Aprile G, Kulikov E, Hill J, Lehle M, Ruschoff J, Kang YK and To GATI: Trastuzumab in combination with chemotherapy versus chemotherapy alone for treatment of HER2-positive advanced gastric or gastro-oesophageal junction cancer (ToGA): A phase 3, open-label, randomised controlled trial. Lancet 376(9742): 687-697, 2010. PMID: 20728210. DOI: 10.1016/S01406736(10)61121-X

7 Hirayama A, Kami K, Sugimoto M, Sugawara M, Toki N, Onozuka H, Kinoshita T, Saito N, Ochiai A, Tomita M, Esumi $\mathrm{H}$ and Soga T: Quantitative metabolome profiling of colon and stomach cancer microenvironment by capillary electrophoresis time-of-flight mass spectrometry. Cancer Res 69(11): 4918-4925, 2009. PMID: 19458066. DOI: 10.1158/0008-5472.CAN-08-4806

8 Yuan LW, Yamashita H and Seto Y: Glucose metabolism in gastric cancer: The cutting-edge. World J Gastroenterol 22(6): 2046-2059, 2016. PMID: 26877609. DOI: 10.3748/wjg.v22.i6.2046

9 Icard P, Shulman S, Farhat D, Steyaert JM, Alifano M and Lincet H: How the Warburg effect supports aggressiveness and drug resistance of cancer cells? Drug Resist Updat 38: 1-11, 2018. PMID: 29857814. DOI: 10.1016/j.drup.2018.03.001

10 Fukushima H, Yasumoto M, Ogasawara S, Akiba J, Kitasato Y, Nakayama M, Naito Y, Ishida Y, Okabe Y, Yasunaga M, Horiuchi H, Sakamoto E, Itadani H, Mizuarai S, Oie S and Yano H: ARHGEF15 overexpression worsens the prognosis in patients with pancreatic ductal adenocarcinoma through enhancing the motility and proliferative activity of the cancer cells. Mol Cancer 15(1): 32, 2016. PMID: 27145964. DOI: 10.1186/s12943-0160516-4

11 Kurose H, Naito Y, Akiba J, Kondo R, Ogasawara S, Kusano H, Sanada S, Abe H, Kakuma T, Ueda K, Igawa T and Yano H: High sulfite oxidase expression could predict postoperative biochemical recurrence in patients with prostate cancer. Med Mol Morphol 52(3): 164-172, 2019. PMID: 30631948. DOI: 10.1007/s00795-018-00214-1

12 Nakamura K, Akiba J, Ogasawara S, Naito Y, Nakayama M, Abe Y, Kusukawa J and Yano H: SUOX is negatively associated with multistep carcinogenesis and proliferation in oral squamous cell carcinoma. Med Mol Morphol 51(2): 102-110, 2018. PMID: 29280012. DOI: 10.1007/s00795-017-0177-4

13 Garrett RM, Johnson JL, Graf TN, Feigenbaum A and Rajagopalan KV: Human sulfite oxidase r160q: Identification of the mutation in a sulfite oxidase-deficient patient and expression and characterization of the mutant enzyme. Proc Natl Acad Sci 
USA 95(11): 6394-6398, 1998. PMID: 9600976. DOI: 10.1073/ pnas.95.11.6394

14 Jin GZ, Yu WL, Dong H, Zhou WP, Gu YJ, Yu H, Yu H, Lu XY, $\mathrm{Xian} \mathrm{ZH}$, Liu YK, Cong WM and Wu MC: SUOX is a promising diagnostic and prognostic biomarker for hepatocellular carcinoma. J Hepatol 59(3): 510-517, 2013. PMID: 23665285. DOI: 10.1016/j.jhep.2013.04.028

15 Japanese Gastric Cancer Association: Japanese Classification of Gastric Carcinoma, 15th ed., Tokyo, Kanehara \& CO. Ltd., 2017.

16 Nakamura K, Sugano H and Takagi K: Carcinoma of the stomach in incipient phase: Its histogenesis and histological appearances. Gan 59(3): 251-258, 1968. PMID: 5726267. DOI: 10.20772/cancersci1959.59.3_251

17 Fukayama M, and Washington MK: WHO Classification of Tumours 5th Edition Digestive System Tumours. World Health Organization, 2019.

18 Nakamura K, Ueyama T, Yao T, Xuan ZX, Ambe K, Adachi Y, Yakeishi Y, Matsukuma A and Enjoji M: Pathology and prognosis of gastric carcinoma. Findings in 10,000 patients who underwent primary gastrectomy. Cancer 70(5): 1030-1037, 1992. PMID: 1515980. DOI: 10.1002/1097-0142(19920901)70:5<1030 ::aid-cncr2820700504>3.0.co;2-c

19 Harrison JD and Fielding JW: Prognostic factors for gastric cancer influencing clinical practice. World J Surg 19(4): 496500, 1995. PMID: 7676690. DOI: 10.1007/bf00294709

20 Wang HM, Huang CM, Zheng CH, Li P, Xie JW, Wang JB, Lin $\mathrm{JX}$ and Lu J: Tumor size as a prognostic factor in patients with advanced gastric cancer in the lower third of the stomach. World J Gastroenterol 18(38): 5470-5475, 2012. PMID: 23082065. DOI: $10.3748 /$ wjg.v18.i38.5470

21 Cancer Genome Atlas Research Network: Comprehensive molecular characterization of gastric adenocarcinoma. Nature 513(7517): 202-209, 2014. PMID: 25079317. DOI: 10.1038/ nature 13480

22 Yamashita K, Sakuramoto S and Watanabe M: Genomic and epigenetic profiles of gastric cancer: Potential diagnostic and therapeutic applications. Surg Today 41(1): 24-38, 2011. PMID: 21191688. DOI: $10.1007 / \mathrm{s} 00595-010-4370-5$
23 Cristescu R, Lee J, Nebozhyn M, Kim KM, Ting JC, Wong SS, Liu J, Yue YG, Wang J, Yu K, Ye XS, Do IG, Liu S, Gong L, Fu J, Jin JG, Choi MG, Sohn TS, Lee JH, Bae JM, Kim ST, Park SH, Sohn I, Jung SH, Tan P, Chen R, Hardwick J, Kang WK, Ayers M, Hongyue D, Reinhard C, Loboda A, Kim S and Aggarwal A: Molecular analysis of gastric cancer identifies subtypes associated with distinct clinical outcomes. Nat Med 21(5): 449-456, 2015. PMID: 25894828. DOI: 10.1038/nm.3850

24 Van Cutsem E, Sagaert X, Topal B, Haustermans K and Prenen H: Gastric cancer. Lancet 388(10060): 2654-2664, 2016. PMID: 27156933. DOI: 10.1016/s0140-6736(16)30354-3

25 Petrelli F, Berenato R, Turati L, Mennitto A, Steccanella F, Caporale M, Dallera P, de Braud F, Pezzica E, Bartolomeo MD, Sgroi G, Mazzaferro V, Pietrantonio F and Barni S: Prognostic value of diffuse versus intestinal histotype in patients with gastric cancer: A systematic review and meta-analysis. J Gastrointest Oncol 8(1): 148-163, 2017. PMID: 8280619. DOI: $10.21037 /$ jgo.2017.01.10

26 Nguyen PH, Giraud J, Chambonnier L, Dubus P, Wittkop L, Belleannee G, Collet D, Soubeyran I, Evrard S, Rousseau B, Senant-Dugot N, Megraud F, Mazurier F and Varon C: Characterization of biomarkers of tumorigenic and chemoresistant cancer stem cells in human gastric carcinoma. Clin Cancer Res 23(6): 1586-1597, 2017. PMID: 27620279. DOI: 10.1158/1078-0432.CCR-15-2157

27 Matoba S: P53 regulates mitochondrial respiration. Science 312(5780): 1650-1653, 2006. PMID: 16728594. DOI: 10.1126/ science. 1126863

28 Luo G, Hu Y, Zhang Z, Wang P, Luo Z, Lin J, Cheng C and Yang Y: Clinicopathologic significance and prognostic value of Ki-67 expression in patients with gastric cancer: A metaanalysis. Oncotarget 8(30): 50273-50283, 2017. PMID: 28488584. DOI: $10.18632 /$ oncotarget.17305

Received September 3, 2020

Revised October 20, 2020

Accepted October 21, 2020 